\title{
Estudio del proceso de enseñanza y aprendizaje a través de las TIC de una institución pública de nivel superior
}

\author{
M.E. Marlene Munguía Martínez \\ marlene_munguia_57@zongolica.tecnm.mx \\ Tecnológico Nacional de México Campus Zongolica \\ Zongolica, Veracruz- México \\ Dra. María Edith Quezada Fadanelli \\ edith_quezada_p40@zongolica.tecnm.mx \\ ORCID ID 0000-0002-1403-9980 \\ Tecnológico Nacional de México Campus Zongolica \\ Zongolica, Veracruz- México \\ M.I.A. Gabriel Ruiz Contreras \\ gabriel_ruiz_ige@zongolica.tecnm.mx \\ ORCID ID 0000-0003-0257-0253 \\ Tecnológico Nacional de México Campus Zongolica \\ Zongolica, Veracruz- México
}

\section{RESUMEN}

En este trabajo se presenta una investigación realizada a estudiantes de educación superior, en una institución pública en el Estado de Veracruz, México, con el objetivo de mostrar el impacto de uso de la tecnología en los procesos de enseñanza y aprendizaje derivado de la pandemia del COVID 19, que intensificó el uso de medios electrónicos en este nivel; para ello se utilizó un cuestionario que fue adaptado al entorno de los estudiantes, compuesto de 13 preguntas cerradas con escala Likert y aplicado mediante un formulario en línea a 422 estudiantes del tercer semestre en adelante, de los tres programas académicos impartidos. Los resultados principales muestran que el $84 \%$ contaba con una computadora, el $98 \%$ con teléfono inteligente y el $79 \%$ con acceso a internet en su hogar para realizar sus actividades académicas; por otro lado, el 69\% afirmó manejar ofimática básica, un 34.83\% identifica información relevante al evaluar fuentes de información, un poco más del $30 \%$ y $25 \%$ respondieron de acuerdo y totalmente de acuerdo, con respecto a una actitud positiva y compromiso ético en el uso de información digital; finalmente más del $50 \%$ se siente satisfecho en el uso de las Tecnologías de Información y Comunicación.

Palabras clave: proceso; enseñanza; aprendizaje; tecnologías de información y comunicación; institución superior. 


\title{
Study of the teaching and learning process through ICT in a public higher education institution
}

\begin{abstract}
This paper presents an investigation conducted with higher education students in a public institution in the State of Veracruz, Mexico, with the objective of showing the impact of the use of technology in the teaching and learning processes derived from the COVID 19 pandemic, which intensified the use of electronic media at this level; for this purpose, a questionnaire was used that was adapted to the students' environment, composed of 13 closed questions with Likert scale and applied through an online form to 422 students from the third semester onwards, from the three academic programs taught. The main results show that $84 \%$ had a computer, $98 \%$ had a smartphone and $79 \%$ had internet access at home to carry out their academic activities; on the other hand, 69\% said they handled basic office automation, $34.83 \%$ identified relevant information when evaluating information sources, a little more than $30 \%$ and $25 \%$ responded in agreement and totally agreed, with respect to a positive attitude and ethical commitment in the use of digital information; finally more than $50 \%$ felt satisfied in the use of Information and Communication Technologies.
\end{abstract}

Keywords: process; teaching; learning; information and communication technologies; higher institution.

Artículo recibido: 05 octubre. 2021 Aceptado para publicación: 02 noviembre 2021 Correspondencia: edith_quezada_p40@zongolica.tecnm.mx Conflictos de Interés: Ninguna que declarar 


\section{INTRODUCCIÓN}

El presente estudio tiene como propósito analizar el uso de las Tecnologías de Información y Comunicación (TIC) en una Institución Pública de Nivel Superior, como medio de apoyo en el proceso de enseñanza y aprendizaje de los alumnos, aunado al incremento en su utilización durante la Pandemia del virus del SARS-CoV 2.

La incorporación de las TIC en el proceso enseñanza y aprendizaje es muy importante en la actualidad, ya que, si se utilizan de manera didáctica, permiten mejorar los resultados académicos y educativos, sin embargo, se debe tener presente que son medios y no el fin mismo del proceso, por lo que su empleo debe estar justificado y su implementación estratégica (Almaguer et al., 2017).

Las habilidades actuales de los estudiantes en el uso de las TIC, en cierta medida, facilitan su aplicación en el proceso, de hecho, las generaciones de hoy, son consideradas "nativos digitales"; no obstante, dichas habilidades son adquiridas de manera informal y condicionadas por diversos factores socioeconómicos, como la posibilidad y disponibilidad de acceso a las TIC, lo cual conlleva a que no todos los estudiantes tengan las mismas condiciones (Daquimela et al., 2019).

En la Educación Superior, el uso de las TIC por parte de la comunidad estudiantil, está enfocado, en el mejor de los casos, en la búsqueda, selección y utilización de información en internet y en el peor de los casos solo es un medio de distracción; por lo que pertenecer a la generación de "nativos digitales", no es sinónimo de un uso correcto de las tecnologías en el proceso de aprendizaje (Pérez et al., 2020).

Dado lo anterior, el presente documento considera, primeramente, el contexto actual de la educación y las TIC. Subsiguientemente, se presenta a las TIC como herramienta importante del aprendizaje.

En los resultados más significativos de los 422 estudiantes encuestados se analizó que el $84 \%$ contaba con una computadora, el $98 \%$ con teléfono inteligente y el $79 \%$ con acceso a internet en su hogar para realizar sus actividades académicas; por otro lado, el 69.09\% afirmó manejar ofimática básica, un 34.83\% identifica información relevante al evaluar fuentes de información, un poco más del $30 \%$ y $25 \%$ de acuerdo y totalmente de acuerdo con respecto a una actitud positiva y compromiso ético en el uso de información digital y manejo de las TIC; finalmente más del 50\% se siente satisfecho en el uso de las TIC. 


\section{EI contexto actual de la educación, habilidades, competencias y las TIC}

En los últimos años el uso de las TIC se ha extendido en el mundo en campos tales como la economía y la educación (Garcés et al., 2016); aunado a ello, la crisis de la pandemia del SARS-CoV 2 (Covid-19) ha catapultado a las TIC en un uso masivo como jamás se había imaginado (Guzmán et al., 2021). En el caso del sistema educativo, ha forzado la migración del ambiente de aprendizaje presencial, a un ambiente mediado por la tecnología, lo cual ha representado un reto para estudiantes, docentes y padres de familia; sin embargo, lo apremiante de su uso hoy en día, no obedece necesariamente a la pandemia actual.

Por ejemplo, en el 2015, los 193 países pertenecientes a las Naciones Unidas, aprobaron un documento denominado "Agenda 2030", el cual contiene 17 objetivos para el desarrollo sostenible; el objetivo 4, "Educación de Calidad" en su meta 4.4 enfatiza que, en el año 2030, los jóvenes y adultos deben alcanzar las competencias adecuadas para mejorar su calidad de vida, mediante trabajos decentes, midiendo dicha meta con la proporción de jóvenes y adultos con competencias en tecnología de la información y las comunicaciones (Comisión Económica para América Latina y el Caribe[CEPAL], 2019). En el caso del Estado de Veracruz, México; el Plan Veracruzano de Desarrollo 20192024, enfatiza en el objetivo 1, respecto a los servicios educativos, que estos deben promover el desarrollo de las habilidades digitales y el uso de Internet con fines pedagógicos (Veracruz Gobierno del Estado [VGE], 2019).

Por lo tanto, es necesario que en todos los niveles educativos se integre de forma creativa y estratégica las TIC en el proceso enseñanza y aprendizaje.

El proceso de enseñanza y aprendizaje a través de las TIC han tenido una detonación y una mayor repercusión en el ámbito educativo, en esta nueva modalidad educativa tecnológica, se puede presentar como ventaja y/o desventajas, generando inconvenientes y desigualdades de aprendizaje.

Por otro lado, cuestionar cuáles son las competencias que debe poseer un docente y un estudiante al utilizar las TIC, deben tener acaso un dominio de diversas disciplinas, competencias pedagógicas para implementar a través de la didáctica estrategias de aprendizaje, para ser capaces de interactuar de manera asertiva y efectiva con el estudiante, por lo cual debemos de ser críticos y autocríticos al momento de reflexionar sobre nuestro quehacer en nuestro contexto actual de la educación, las habilidades y 
competencias.

En este contexto educativo, las competencias docentes son comprendidas como el conjunto de conocimientos, habilidades (destrezas) y actitudes (desempeño, motivación, valores, intereses, características entre otros componentes personales, sociales, etc.), que favorecen a los docentes de manera integral para resolver de forma eficiente las situaciones a las que se enfrentan en su quehacer académico, pedagógico y profesional (Hernández, Gamboa \& Ayala, 2014).

Las competencias académicas del estudiante son todo lo que debe saber, conocer y que está relacionado con las disciplinas particulares y su práctica significativa para crear, innovar, generar nuevos conocimientos y aprendizaje de lo adquirido.

De acuerdo a Gamboa (2016) donde se considera de acuerdo al ámbito académico, la formación tecnológica y las competencias pedagógicas sobre el uso de las TIC, permiten a los docentes favorecer su práctica académica y por ende de manera simultánea el desarrollo de competencias por parte de los estudiantes. Sin embargo, muchos docentes están en la transición digital, lo que significa que operan y es parte de su dinámica el uso de la tecnología con regularidad con fines instrumentales (profesionales, personales, sociales, etc.), pero aun así, siempre es requerido elevar el nivel de competencia en el uso de las TIC para que su empleo proporcione resultados dentro y fuera del aula.

Las TIC de acuerdo a Gilbert et al., (1992, p.1), donde hace mención al "conjunto de herramientas, soportes y canales para el tratamiento y acceso a la información"., definiéndose como herramientas que se utilizan como canales para el proceso y distribución de la información. Para Bartolomé (1989, p.11) señala que se refiere a los últimos desarrollos tecnológicos e innovaciones y sus aplicaciones. Así como elementos más representativos de las nuevas tecnologías. Si bien para Santillana de Tecnología Educativa (1991), donde las describe como los últimos desarrollos de la tecnología de la información que en nuestros días se identifican por su constante innovación, sin duda el ordenador más específico de la era digital.

En esta misma línea el diccionario de "Castells et al., (1986) hace mención que se comprenden una serie de aplicaciones de descubrimiento científico, cuyo núcleo central consiste en una capacidad cada vez mayor de procesamiento de la información". Y por último el concepto publicado en la revista "Cultura y Nuevas Tecnologías" de la Exposición Procesos, que lo describe como “... nuevos soportes y canales para dar forma, 
registrar, almacenar y difundir contenidos informacionales." (Ministerio de Cultura, 1986, p.12). Donde se amplían considerablemente las posibilidades de acceso, producción de información en diferentes códigos.

El término habilidades ha sido ampliamente definido Según la Real Academia Española (RAE), se comprende como las habilidades y competencias, así como la capacidad que tiene una persona para desempeñarse de manera correcta y con facilidad para realizar tareas o actividades específicas. De esta manera, se trata de una forma de aptitudes específica y especializadas para una actividad puntual, sea de índole físico, cognitiva o social.

Para Díaz Barriga y Rigo (2000) donde convergen la educación y las TIC, hacia un nuevo paradigma de tecnología, innovación y educación, indica en su concepto de competencia donde hace referencia a un saber hacer de manera eficiente, demostrable mediante desempeños observables:

Donde trata de una capacidad para resolver problemas que se aplica de manera flexible y pertinente, adaptándose a las nuevas tecnologías de la información y comunicación, herramienta que interviene en las actividades laborales, académicas, atendiendo a las demandas que plantean situaciones diversas, desde la óptica de los promotores de la EBC, la competencia no se limita a los aspectos procedimentales del conocimiento, a la mera posesión de habilidades y destrezas, sino que se ve acompañada necesariamente de elementos teóricos y actitudinales. p. 79

Para Tobón (2006) las competencias son: "procesos complejos de desempeño con idoneidad en un determinado contexto, con responsabilidad", dicha referencia implica:

Operaciones, transacciones, producción de información, pasos que ocurre día con día dentro y fuera del ámbito educativo. La referencia a procesos, considerados como aquellas acciones con un inicio y un fin identificables, que cumplen con determinados objetivos, metas o demandas del contexto, por lo que las competencias no son estáticas, sino dinámicas, creativas, adaptativas al contexto, necesidades dadas las características y demandas del ámbito de desempeño. p.5 
Sin embargo, uno de los factores determinantes es la forma en que se lleva a cabo el proceso de enseñanza y aprendizaje a través de las TIC, es la medición de las habilidades y competencias, ya que cada institución trata de crear, diseñar un sistema que involucre e incluya a todos los sistemas superiores del país, para así lograr que el Índice General de Habilidades y Competencia sea lo más uniforme, estándar posible y que los niveles sean equivalentes de una institución a otra. Así mismo, el común denominador en las diferentes aproximaciones a este análisis es la educación especializada, infraestructura, la innovación y tecnológica.

Considerando que la formación superior especializada genera personas competitivas, con alto potencial, con un nivel de calidad instruccional, relacionándose así con el progreso y bienestar.

La infraestructura generadora de ambientes óptimos para el desarrollo y desempeño de las actividades industriales, económicas, sociales y culturales, etc. La innovación genera cambios de forma creativa, variaciones en los ámbitos, contextos o productos, generando una transformación y la tecnología siendo un medio de adaptación e inclusión de los sectores para representar el aumento de la productividad.

Las TIC no solamente están exigiendo un cambio a profundidad el significado de la educación sino que además se han constituido como una de las herramientas para adaptarse a los contextos, ámbitos. Al cuestionarnos acerca de lo que es un entorno innovador en el ámbito del aprendizaje seguramente nos viene a la mente un salón de clases en donde se utilizan las TIC; pero hay que tener una perspectiva más amplia e integral, ya que suele existir la brecha digital-tecnológica, la que viven los estudiantes dentro y fuera de la institución, ya que los estudiantes han desarrollados las habilidades y competencias de forma empíricas, enfocándose más a la comunicación entre pares que a favorecer un pensamiento crítico, independiente y creativo, esto no es suficiente para transitar hacia el uso de las nuevas tecnologías como herramienta de apoyo y facilitador del proceso de enseñanza y aprendizaje.

Es prioritario que el ámbito educativo se involucre en la transformación integral, con herramientas óptimas (materiales y recurso humano), promoviendo, aplicando en las asignaturas la incorporación de las TIC, para el desarrollo de habilidades, competencias y conocimientos prioritarios para nuevas enseñanzas del docente y aprendizajes para los estudiantes, para poder ser parte de la cultura digital. 


\section{ESTRATEGIAS METODOLÓGICAS O MATERIALES Y MÉTODOS}

La investigación fue descriptiva se realizó en dos fases, se buscó mostrar el impacto del uso de la tecnología en los procesos de enseñanza aprendizaje, ya que a raíz de la pandemia del COVID 19 se intensificó el uso de medios electrónicos para la educación superior.

En la primera fase se hizo una revisión de la literatura para identificar el contexto general de las TIC en la educación superior, se tomó de referencia un cuestionario que fue adaptado al entorno de los estudiantes denominado "Uso de las TIC por estudiantes de educación superior" compuesto de 13 preguntas cerradas; en las cuales, como respuesta se solicitaba un grado de acuerdo o desacuerdo, según la escala likert (Plascencia \& Beltrán, 2016).

En la segunda fase se realizó un estudio descriptivo transversal, se aplicó el cuestionario a través de Google Forms a estudiantes de educación superior, inscritos al semestre septiembre 2020-enero 2021 en el Tecnológico Nacional de México Campus Zongolica en la sede localizada en Nogales, Veracruz, México.

El instrumento se suministró a los alumnos de los tres programas académicos ofertados en el campus en ese periodo, esto es, Ingeniería en Gestión Empresarial, Ingeniería en Sistemas Computacionales e Ingeniería en Desarrollo Comunitario, donde la condición principal fue que los estudiantes al menos tuvieran un año escolar cursado y aprobado, es decir que no fueran de nuevo ingreso. La muestra total de cuestionarios aplicados fue de 422 estudiantes, 195 de Ingeniería en Gestión Empresarial, 145 de Ingeniería en Sistemas Computacionales y 82 de Ingeniería en Desarrollo Comunitario. Se obtuvieron datos de las condiciones tecnológicas y de innovación con las que cuentan los alumnos.

El cuestionario fue validado a través de una prueba piloto y la confiabilidad del instrumento fue calculada mediante el coeficiente de consistencia interna de Cronbach, que se muestra en la Tabla 1:

Tabla 1. Estadísticas de fiabilidad

\begin{tabular}{ll}
\hline Alfa de Cronbach $\quad$ Número de elementos \\
\hline
\end{tabular}

$0.983 \quad 13$

Fuente: Elaboración propia con base en el software SPSS versión 22 con datos recabados en el 2020. 
Como lo menciona Ruiz (2002) que un valor en un rango de 0.81 a 1.00 , muy alta; 0.61 a 0.80 , alta; 0.41 a 0.60 , moderada; 0.21 a 0.40 , baja y 0.01 a 0.20 , muy baja. El valor obtenido del coeficiente fue de 0.983 por tanto es considerado muy alto.

\section{RESULTADOS Y DISCUSIÓN}

De la muestra analizada se encontró que de los tres programas académicos estudiados en la Ingeniería en Gestión Empresarial predominan las mujeres, en lo concerniente a la Ingeniería en Sistemas Computacionales hay mayoría de hombres y en la Ingeniería en Desarrollo Comunitario hay igual número de hombres y mujeres. La edad oscila entre 18 y 20 años, el $82 \%$ de los encuestados cursan el tercer semestre mientras el 18\% están en el quinto semestre.

Uno de los puntos evaluados fue si contaban con una computadora para realizar sus actividades académicas, el 84\% de los alumnos afirmaron tenerla y sólo el 16\% mencionó que no contaba con este recurso. En cuanto al dispositivo móvil que poseen, el $98 \%$ mencionó contar con un teléfono celular, mismo que utilizan para navegar en internet y así obtener información para realizar las actividades de sus respectivos programas.

El 79\% de los estudiantes comentó que tiene acceso diario a internet el cual lo hacen desde sus casas, el $21 \%$ afirmó que no tenían acceso a internet en sus hogares y ocasionalmente lo hacen en sitios públicos o acuden a rentar el servicio a un ciber café.

En la segunda parte del instrumento administrado, se encontró que el 62.09\% de los estudiantes afirman utilizar, además de computadoras, diferentes dispositivos electrónicos (teléfonos inteligentes, tabletas, entre otros) para realizar trabajos académicos (31.28\% de acuerdo y el $30.81 \%$ totalmente de acuerdo).

Del mismo modo, el $62.09 \%$ de estudiantes afirmó manejar herramientas ofimáticas ( $28.67 \%$ totalmente de acuerdo y $33.41 \%$ de acuerdo). Sin embargo, menos de la mitad de los estudiantes encuestados (20.62\% totalmente de acuerdo y $26.78 \%$ de acuerdo) afirmaron el uso de organizadores gráficos y programas para representar las relaciones entre ideas y conceptos.

Por otra parte, en relación a la localización de información a través de diferentes sitios Web y demás contenidos en Internet (videos, blogs, audios, entre otros), el $32.46 \%$ de los estudiantes contestó estar totalmente de acuerdo. En este mismo sentido, el $34.83 \%$ de los estudiantes afirmaron estar de acuerdo en identificar la información relevante al evaluar las distintas fuentes y la procedencia de las mismas. 
Además, los estudiantes manifestaron que tienen una actitud positiva hacia el uso de las TIC para apoyar el aprendizaje, la colaboración y la productividad (33.41 \% de acuerdo y $27.96 \%$ totalmente de acuerdo).

Otro aspecto relevante que se encontró es que los encuestados manifestaron asumir un compromiso ético en el uso de la información digital y de las TIC, respetar los derechos de autor, propiedad intelectual y documentación adecuada de las fuentes utilizadas (32.70\% de acuerdo y $28.67 \%$ totalmente de acuerdo).

Finalmente, como se puede observar en la figura 1 los estudiantes de la institución de educación superior pública analizada, en este caso el Tecnológico Nacional de México Campus Zongolica Sede Nogales tienen en general más del 50\% de satisfacción en el uso de las TIC.

\section{Figura 1}

\section{Grado de satisfacción en el uso de las TIC}

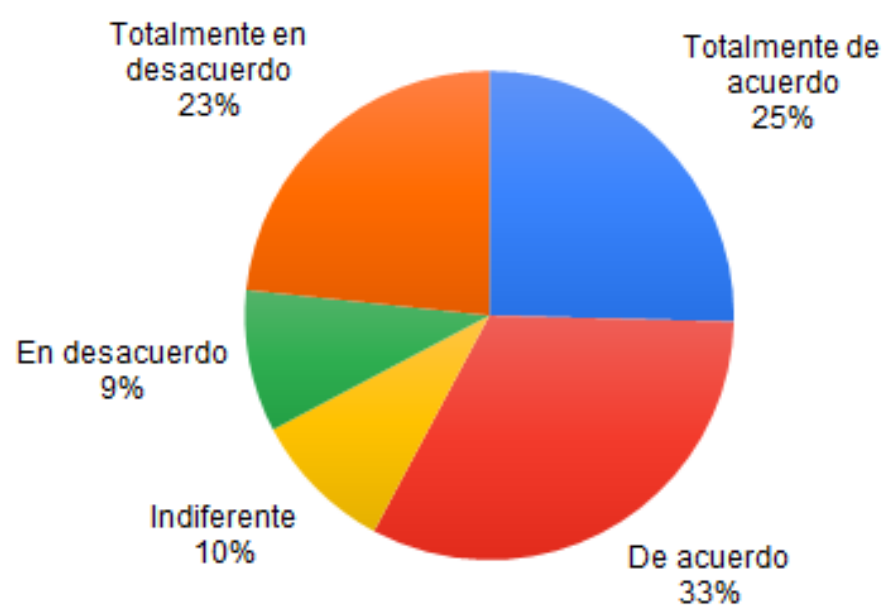

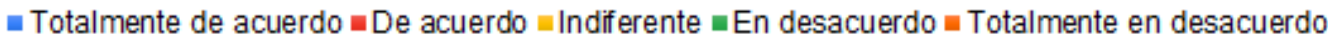

Fuente: Elaboración propia en base al cuestionario aplicado a los alumnos del ITSZ en septiembre 2020 - enero 2021

Así mismo, se puede destacar que en el sistema de enseñanza aprendizaje tienen un rol significativo las TIC como soporte en la interacción con las tareas didácticas que integran diversas competencias digitales que facilitan el proceso de educación con una gama de herramientas para que el docente interactúe con los estudiantes (García-Sánchez, Reyes -Añorve y Godínez-Alarcón, 2018). 


\section{CONCLUSIÓN O CONSIDERACIONES FINALES}

En este estudio se mostró que más del 50 por ciento de los estudiantes están satisfechos con la utilización de las TIC como parte de sus actividades de aprendizaje; adicionalmente la mayoría de ellos cuentan con teléfonos celulares, mismo que utilizan para navegar en internet y así obtener información para realizar las actividades académicas.

También se pudo evidenciar que más del 60 por ciento de ellos manejaba herramientas ofimáticas; sin embargo, menos de la mitad de los estudiantes afirmaron el uso de organizadores gráficos y programas para manejo de información.

Así mismo, se pudo identificar que los alumnos no dominan las habilidades de localización de información en la red, de esto derivan algunas deficiencias a la hora de documentar sus escritos académicos.

Otro punto importante a destacar de este estudio fue la actitud positiva que manifestaron los estudiantes hacia el uso de las TIC para apoyar su aprendizaje, la colaboración y la productividad.

Adicionalmente, los encuestados manifestaron tener un compromiso ético con el uso de la información digital y de las TIC, respetar los derechos de autor, propiedad intelectual y dar crédito de las fuentes utilizadas.

Por otra parte, en un estudio denominado "La Educación superior durante la contingencia sanitaria COVID-19: Uso de las TIC como herramientas de aprendizaje. Caso de estudio: alumnos de la Facultad de Contaduría y Administración en la Universidad Autónoma de Chihuahua" se encontró que había una correlación positiva hacía las TIC, que se mostró en los diferentes aspectos examinados: estilo de aprendizaje, dominio en el uso de las TIC, redes sociales, programa educativo, nivel de satisfacción de contar con los recursos tecnológicos apropiados, entre otras; en todas ellas se manifiesta una predisposición favorable; se puede decir que hay una tendencia positiva hacia el uso de las TIC de igual forma que se puede identificar en los estudiantes que participaron en el estudio de la institución pública analizada (Sapién-Aguilar et al., 2020).

De acuerdo a Cabero (2017), las TIC y el acceso a dispositivos tecnológicos son indispensables para un entorno globalizado, recursos que han resaltado su importancia en la crisis por el COVID-19, donde el aprendizaje remoto ha sido una opción para dar continuidad a la educación, siendo una necesidad desarrollar tanto en docentes como alumnos las competencias digitales. 
Sin embargo, es importante considerar la brecha digital y la dificultad para acceder a las tecnologías ha influido para que muchos estudiantes que carecen de recursos puedan acceder a computadoras o no tienen conectividad a su disposición, lo cual impacta en el incremento de la tasa de abandono en las aulas, situación que prevalece en escuelas públicas donde los alumnos tienen condiciones económicas precarias en comparación con los estudiantes de escuelas privadas (Banco Interamericano de Desarrollo[BID], 2020). Como recomendación se podría decir que la pandemia aceleró el uso de las TIC en muchas actividades cotidianas y la educación no fue la excepción, tanto docentes como estudiantes tuvieron que desarrollar habilidades en el proceso de enseñanza aprendizaje, una crisis que nadie estaba preparado para afrontarla, sin embargo el uso de las TIC ha sido una herramienta fundamental para continuar con el gran reto de la educación a distancia, no obstante se requiere de un proceso continuo de capacitación de los docentes y a su vez de los alumnos para aprovechar al máximo las bondades que ofrecen las tecnologías de la información.

\section{REFERENCIAS BIBLIOGRAFICAS}

Adell, J. (1997). Tendencias en Educación en la Sociedad de las Tecnologías de la Información., en Revista EDUTEC, 7 disponible en http:// www.uib.es/depart/gte/revelec.html [consultado septiembre 27, 2021].

Almaguer, A. D., Zaquinaula, F. P., Ramírez, T. M., \& Martínez, I. R. (2017). Las Tic En El Proceso De Enseñanza Aprendizaje. Obtenido de European Scientific Journal ESJ, 13(34), 269: https://doi.org/10.19044/esj.2017.v13n34p269

Banco Interamericano de Desarrollo. (2020). La educación Superior en tiempos de COVID-19. Nueva York: BID.

Cabero, J. (2017). La formación en la era digital: ambientes enriquecidos por la tecnología - Dialnet. Revista de Gestión de La Innovación, 2(1), 34-53. https://doi.org/ISSN 0719-742X E-ISSN 0719-7624

Comisión Económica para América Latina y el Caribe (CEPAL). (2019). La Agenda 2030 y los Objetivos de desarrollo sostenible. Una oportunidad para América Latina y el Caribe. Obtenido de https://repositorio.cepal.org/bitstream/handle/11362/40155/24/S1801141_es.pdf

Daquimela, B. A., Benítez, C. R., \& Jamarillo, J. A. (2019). Desarrollo de las habilidades TIC en los estudiantes. Obtenido de Sociedad \&Amp; Tecnología, 2(2), 36-44: 
https://doi.org/10.51247/st.v2i2.48

Díaz Barriga, F. y Rigo, M. (2000). "Formación docente y educación basada en competencias", en M. A. Valle Formación en competencias y certificación profesional (pp. 76-104). México: Universidad Nacional Autónoma de México. http://132.248.192.241/ editorial/wp-

content/uploads/2018/06/Formaci\%C3\%B3n\%20en\%20competencias\%20y\%20 certificaci\%C3\%B3n\%20profesional.pdf [consultado septiembre 27, 2021]

ESTEVE, F. (2014). La competencia digital docente: más allá de las habilidades TIC. Recuperado de: http://www.francescesteve.es/la-competencia-digital-docentemas-alla-de-las-habilidades-tic/

FERNÁNDEZ De La IGLESIA, J. (2012). Competencias TIC de los docentes para la sociedad del conocimiento. Tesis doctoral. Recuperado de: http://dspace.usc.es/handle/10347/6100

García Sánchez, M. del R., Reyes Añorve, J., \& Godínez Alarcón, G. (2018). Las Tic en la educación superior, innovaciones y retos / The ICT in higher education, innovations and challenges. RICSH Revista Iberoamericana De Las Ciencias $\begin{array}{lllll}\text { Sociales } \quad Y \quad \text { Humanísticas, } & 6(12), & 299 \quad \text { - } & 316 .\end{array}$ https://doi.org/10.23913/ricsh.v6i12.135

García-Valcárcel A., (1998) La actitud de los futuros maestros hacia las Nuevas Tecnologías [Revista Electrónica de Tecnología Educativa] Edutec 97 Disponible en http//.www.ieev.uma.es/ edutec97/edu97_c3/2-3-13.htm [consultado septiembre 27, 2021].

Garcés Suárez Elizabeth, Garcés Suárez, Emma, \& Alcívar Fajardo, Orly. (2016). LAS TECNOLOGÍAS DE LA INFORMACIÓN EN EL CAMBIO DE LA EDUCACIÓN SUPERIOR EN EL SIGLO XXI: REFLEXIONES PARA LA PRÁCTICA. Revista Universidad y Sociedad, 8(4), 171-177. Recuperado en 04 de noviembre de 2021, de http://scielo.sld.cu/scielo.php?script=sci_arttext\&pid=S221836202016000400023\&lng=es\&tlng=es.

Guzmán de Castro, B. J., Castro, S., \& Acosta Cruz, W. D. (2021). TIC y educación en tiempos difíciles. Delectus, 4(2), 1-11. https://doi.org/10.36996/delectus.v4i2.117

HERNÁNDEZ, s. c., arévalo d. M., GAMBOA, S. A. (2016). COMPETENCIAS TIC PARA EL DESARROLLO PROFESIONAL DOCENTE EN EDUCACIÓN 
BÁSICA.

OBTENIDO

$\mathrm{DE}$

https://www.redalyc.org/journal/4772/477249927002/html/

HERNANDEZ, C., GAMBOA, A., \& AYALA, E. (2014). Competencias TIC para los docentes de educación superior. Congreso Iberoamericano de Ciencia, Tecnología, Innovación y Educación. Buenos Aires. Recuperado de: http://www.oei.es/congreso2014/memoriactei/837.pdf

Pérez, F. L., Bas, M. C., \& Nahón, A. E. (2020). Autopercepción sobre habilidades digitales emergentes en estudiantes de Educación Superior. Obtenido de Comunicar: Revista científica iberoamericana de comunicación y educación, (62), 91-101: https://doi.org/10.3916/C62-2020-08

Real Academia Española. (s.f.). Cultura. En Diccionario de la lengua española. Recuperado SEPTIEMBRE 27, 2021, de https://dle.rae.es/habilidad

Ruiz Bolívar, C. (2002). Instrumentos de Investigación Educativa.

Venezuela: Fedupel.

Sapién Aguilar, A. L., Piñón Howlet, L. C., Gutiérrez-Diez, M. C. y Bordas Beltrán, J. L. (2020).La Educación superior durante la contingencia sanitaria COVID-19: Uso de las TIC como herramientas de aprendizaje. Caso de estudio: alumnos de la Facultad de Contaduría y Administración. Revista Latina de Comunicación Social, 78, 309-328.https://www.doi.org/10.4185/RLCS-2020-1479

Tobón, S. (2006). Aspectos básicos de la formación basada en competencias. Talca: Proyecto Mesesup. Consultado SEPTIEMBRE 27, 2021 en: file:///D:/Descargas/22.pdf

UNESCO (2008). Estándares de competencias en TIC para docentes. Recuperado de: http://www.eduteka.org/EstandaresDocentesUnesco.php

Veracruz Gobierno del Estado (VGE). (2019). Plan Veracruzano de Desarrollo 2019 2024. Obtenido de http://www.veracruz.gob.mx/programadegobierno/2019/06/06/planveracruzano-de-desarrollo-2019-2024 